IDDF2020-ABS-0053 PREDICTION OF PATIENT MORTALITY RELATED TO THE LIVER DYSFUNCTION IN ICU BASED ON MACHINE LEARNING

Yi Yu*. Department of Critical Care Medicine, The Second Affiliated Hospital of Guangzhou University of Chinese Medicine, China

\subsection{6/gutjnl-2020-IDDF.139}

Background The traditional mortality prediction method for patients related to the liver dysfunction is to use the APACHE scoring system for mortality prediction. To evaluate the prediction of patient mortality related to the liver dysfunction in ICU based on machine learning.

Methods It directly extracts patient data from the MIMIC-III and Philips eICU collaboration databases. For the missing value part of the sample set, we analyzed the significance of sample characteristics Gini, chose to use the median to fill missing values on the MIMIC-III sample set, and use the majority to fill missing values on the eICU sample set. After normalizing the data, principal component analysis (PCA) was used to reduce the dimensionality of the sample set's sample features, and the effect of the predictive model trained on the sample sets before and after dimension reduction was compared. Next, we used machine learning methods such as random forest, support vector classification, and multi-layer perceptron neural network to achieve liver dysfunction prediction, mortality prediction, and liver dysfunction on the MIMIC-III and eICU sample sets, respectively.

Results In the process of analyzing the predictive model, we found that some of the sample features showed higher importance in the prediction of patient mortality by comparing the Gini importance of sample features. At the same time, compared with the original sample set, the anion gap shows a higher Gini importance when predicting the mortality of the liver dysfunction sample set, and the liver dysfunction sample set after removing the anion gap. The rate of death predictions has slipped, suggesting a potential link between anion gaps and the liver dysfunction.

Conclusions Compared with the original sample set, the lactic acid shows a higher Gini importance when predicting the mortality of liver dysfunction sample set, and the liver dysfunction sample set after removing the lactic acid. The rate of death predictions has slipped, suggesting a potential link between lactic acid and liver dysfunction.

\section{IDDF2020-ABS-0057 RISK OF INCIDENT HEPATOCELLULAR CARCINOMA (HCC) IN CHRONIC HEPATITIS B (CHB) VIRUS-INFECTED PATIENTS TREATED WITH TENOFOVIR DISOPROXIL FUMARATE (TDF) VERSUS ENTECAVIR (ETV): A US ADMINISTRATIVE CLAIMS ANALYSIS}

${ }^{1}$ Ray Kim*, ${ }^{2}$ Laura Telep, ${ }^{3}$ Mei Lu, ${ }^{2}$ Heribert Ramroth, ${ }^{2}$ John Flaherty, ${ }^{2}$ Anuj Gaggar, ${ }^{2}$ Anand Chokkalingam, ${ }^{2}$ Carol Yee Kwan Chan, ${ }^{3}$ Stuart Gordon. ${ }^{1}$ Stanford University, Palo Alto, CA, USA; ${ }^{2}$ Gilead Sciences, Foster City, CA, USA; ${ }^{3}$ Henry Ford Health System, Detroit, USA

\subsection{6/gutjnl-2020-IDDF.140}

Background TDF and ETV are first-line treatments for CHB virus infection. A recent analysis of nationwide insurance data from Korea suggested that the risk of HCC may vary by type of $\mathrm{CHB}$ treatment; however, this finding was based on a limited follow-up period following TDF approval in Korea in 2012 and has not been replicated in large datasets outside of Asia. The objective of this analysis was to explore the longterm risk of HCC with TDF vs. ETV in treatment naïve (TN) $\mathrm{CHB}$ patients in a US administrative claims dataset.

Methods Among 158,272 patients with evidence of CHB and administrative medical claims between January 2006 and September 2018, we identified TN patients exposed to TDF $(\mathrm{N}=6,145)$ or ETV $(\mathrm{N}=4,046)$ with at least 1 year of continuous enrollment prior to cohort entry. Exclusion criteria included coinfection with $\mathrm{HCV}, \mathrm{HDV}$, or HIV; prior exposure to peginterferon or nucleos(t)ide analogues; HCC or liver transplant prior or up to 6 months after initiating treatment. Absolute rates and corresponding 95\% CIs were determined for each treatment group, Cox proportional hazards methods were used to estimate the risk of incident HCC associated with TDF vs ETV. To account for the effects of potential differences in demographics and baseline health status, we incorporated multivariable adjustment and weighting based on treatment propensity scores.

Results Median follow-up duration was comparable in TDFvs ETV-treated patients among TDF-treated CHB patients, the absolute rate of HCC was approximately half that of patients treated with ETV (0.32 per 100 PY (CI: 0.23 - 0.43) vs 0.61 per 100 PY (CI: $0.45-0.80)$ ). After adjustment for age group, sex, baseline health conditions and propensity score weighting, TDF remained associated with a significantly decreased risk of HCC compared to treatment with ETV (HR: 0.56, CI: $0.37-0.86$ ).

Conclusions In this analysis of commercially insured, TN CHB patients in the US, the absolute rate of HCC was lower in those treated with TDF than with ETV. After adjustment, treatment with TDF remained associated with a significantly decreased long term risk of HCC, consistent with recent findings from Asia.

\section{IDDF2020-ABS-0058 48-WEEK SAFETY AND EFFICACY OF SWITCHING TO TENOFOVIR ALAFENAMIDE (TAF) FROM TENOFOVIR DISOPROXIL FUMARATE (TDF) IN CHRONIC HBV ASIAN PATIENTS WITH TDF RISK FACTORS (RF)}

${ }^{1}$ Sang Hoon Ahn*, ${ }^{2}$ Jia-Horng Kao, ${ }^{3}$ Hie-Won Hann, ${ }^{4}$ Fung Scott, ${ }^{5}$ Huy Trinh, ${ }^{6}$ Tuan Trong Nguyen, ${ }^{7}$ Seung Woon Paik, ${ }^{8}$ Anuj Gaggar, ${ }^{8}$ John Flaherty, ${ }^{8}$ Leland Yee, ${ }^{8}$ Belinda Jump, ${ }^{8}$ Shalini Sethi, ${ }^{8}$ George Wu, ${ }^{8}$ Carol Yee Kwan Chan. ${ }^{1}$ Severance Hospital, Korea, South; ${ }^{2}$ Department of Internal Medicine, National Taiwan University Hospital, Taiwan; Hepatitis Research Center, National Taiwan University Hospital, Taiwan; Graduate Institute of Clinical Medicine, National Taiwan University College of Medicine, Taiwan; ${ }^{3}$ Thomas Jefferson University Hospital, Philadelphia, USA; ${ }^{4}$ Toronto Centre for Liver Disease, Toronto General Hospital, University Health Network, Canada; ${ }^{5}$ San Jose Gastroenterology, San Jose, USA; ${ }^{6}$ Research and Education, Inc.,San Diego, USA; ${ }^{7}$ Department of Internal Medicine, Samsung Medical Center, Sungkyunkwan University School of Medicine, Korea, South; ${ }^{8}$ Gilead Sciences, Foster City, USA

\subsection{6/gutjnl-2020-IDDF.141}

Background In a recent Phase 3 study (Study 4018) in HBV patients suppressed on TDF treatment, switching to TAF demonstrated non-inferior efficacy to continued TDF with superior bone and renal safety at Week 48. This study is to assess the safety and efficacy of switching to TAF from TDF in patients of Asian descent with risk factors for TDF toxicity as per current EASL and AASLD guidelines. 